Mathematical Modelling and AnAlysis

Volume 17 Number 2, April 2012, 271-280

http://dx.doi.org/10.3846/13926292.2012.662705

(c) Vilnius Gediminas Technical University, 2012
Publisher: Taylor\&Francis and VGTU

http://www.tandfonline.com/TMMA

Print ISSN: 1392-6292

Online ISSN: 1648-3510

\title{
On Discrete Universality of Composite Functions
}

\section{Jovita Rašytè}

Vilnius University

Naugarduko 24, LT-03225 Vilnius, Lithuania

E-mail: jovita.ras@gmail.com

Received September 26, 2011; revised January 22, 2012; published online April 1, 2012

Abstract. In 1975, S.M. Voronin proved that the Riemann zeta-function $\zeta(s)$ is universal in the sense that its shifts approximate uniformly on some sets any analytic function. Let $h$ be a fixed positive number such that $\exp \left\{\frac{2 \pi k}{h}\right\}$ is irrational for all $k \in \mathbb{Z} \backslash\{0\}$. In the paper, the classes of functions $F$ such that the shifts $F(\zeta(s+i m h))$, $m \in \mathbb{N} \cup\{0\}$, approximate any analytic function are presented. For the proof of theorems, some elements of the space of analytic functions are applied.

Keywords: Riemann zeta-function, support of a measure, space of analytic functions, universality.

AMS Subject Classification: 11M06.

\section{Introduction}

Let $s=\sigma+i t$ denote a complex variable. The Riemann zeta-function $\zeta(s)$ is defined, for $\sigma>1$, by the Dirichlet series

$$
\zeta(s)=\sum_{m=1}^{\infty} \frac{1}{m^{s}},
$$

and is analytically continuable to the whole complex plane, except for a simple pole at $s=1$ with residue 1 . Moreover, the function $\zeta(s)$, for $\sigma>1$, can be written as the Euler product over primes, namely,

$$
\zeta(s)=\prod_{p}\left(1-\frac{1}{p^{s}}\right)^{-1}
$$

In 1975, S.M. Voronin discovered [11] a remarkable universality property of the function $\zeta(s)$. Roughly speaking, he proved that every analytic function can be approximated with a given accuracy uniformly on some sets of the strip $D=\left\{s \in \mathbb{C}: \frac{1}{2}<\sigma<1\right\}$ by shifts $\zeta(s+i \tau), \tau \in \mathbb{R}$. A modern version of the Voronin theorem has the following form [6]. We will denote by meas $\{A\}$ the Lebesgue measure of a measurable set $A \subset \mathbb{R}$. 
Theorem 1. Suppose that $K$ is a compact subset of the strip $D$ with connected complement, and $f(s)$ is a continuous non-vanishing function on $K$ which is analytic in the interior of $K$. Then, for every $\varepsilon>0$,

$$
\liminf _{T \rightarrow \infty} \frac{1}{T} \operatorname{meas}\left\{\tau \in[0, T]: \sup _{s \in K}|\zeta(s+i \tau)-f(s)|<\varepsilon\right\}>0 .
$$

The inequality of Theorem 1 shows that the set of shifts $\zeta(s+i \tau)$ approximating a given analytic function is sufficiently wide: it has a positive lower density.

A. Reich observed [9], see also [10], that another, discrete, universality of the function $\zeta(s)$ is possible. In this case, $\tau$ in the shifts $\zeta(s+i \tau)$ runs not the interval $[0, T]$ but some discrete set, for example, takes values in an arithmetic progression.

Let $h>0$ be a fixed number. We will limit ourselves by the case when the number $\exp \left\{\frac{2 \pi k}{h}\right\}$ is irrational for all $k \in \mathbb{Z} \backslash\{0\}$.

Theorem 2. Let the set $K$ and function $f(s)$ satisfy the hypotheses of Theorem 1. Then, for every $\varepsilon>0$,

$$
\liminf _{N \rightarrow \infty} \frac{1}{N+1} \sharp\left\{0 \leq m \leq N: \sup _{s \in K}|\zeta(s+i m h)-f(s)|<\varepsilon\right\}>0 .
$$

Theorem 2 under stronger hypotheses on the set $K$ has been obtained in [2], however, it is easy to replace these hypotheses by those of Theorem 2 .

The initial Voronin's proof of the universality theorem for the Riemann zetafunction is based on the analogue of the Riemann theorem on rearrangement of terms in series in Hilbert space [8]. Also, another approach using probabilistic limit theorems in the space of analytic functions for the proof of universality theorems is known $[2,6,10]$. Both these approaches are examples of application of functional analysis.

It is not difficult to see that some functions of $\zeta(s)$ also preserve the universality property. Define $\log \zeta(s)$ in the strip $D$ by continuous variation from $\log \zeta(2) \in \mathbb{R}$ along the line segments $[2,2+i t]$ and $[2+i t, \sigma+i t]$ provided that the path does not pass a possible zero or pole $s=1$. If this does, then we take

$$
\log \zeta(\sigma+i t)=\lim _{\epsilon \rightarrow+0} \log \zeta(\sigma+i(t+\varepsilon)) .
$$

Then Theorems 1 and 2 hold for $\log \zeta(s)$, however, in this case, the requirement that $f(s)$ is non-vanishing on $K$ is not needed. By the way, in [1], the universality for the function $\zeta(s)$ is derived from that of $\log \zeta(s)$. Also, a simple application of the integral Cauchy formula leads to the universality of the derivative $\zeta^{\prime}(s)$ with the same remark on the non-vanishing of an approximated function. Therefore, a problem arises to describe some classes of functions $F$ such that the composite function $F(\zeta(s))$ remains universal in the above sense. In the case of Theorem 1, this was done in [7]. The aim of this paper is to prove that the propery of discrete universality is valid for composite functions $F(\zeta(s))$.

Let $G$ be a region on the complex plane. Let denote by $H(G)$ the space of analytic functions on $G$ equipped with the topology of uniform convergence on 
compacta. The space $H(G)$ is metrizable. It is well known, see, for example, [4], that there exists a sequence of compact subsets $\left\{K_{l}: l \in \mathbb{N}\right\} \subset G$ such that $G=\bigcup_{l=1}^{\infty} K_{l}, K_{l} \subset K_{l+1}$ for all $l \in \mathbb{N}$, and if $K \subset G$ is a compact subset, then $K \subset K_{l}$ for some $l \in \mathbb{N}$. Define

$$
\rho\left(g_{1}, g_{2}\right)=\sum_{l=1}^{\infty} 2^{-l} \frac{\sup _{s \in K_{l}}\left|g_{1}(s)-g_{2}(s)\right|}{1+\sup _{s \in K_{l}}\left|g_{1}(s)-g_{2}(s)\right|}, \quad g_{1}, g_{2} \in H(G) .
$$

Then $\rho$ is a metric in the space $H(G)$ which induces the topology of uniform convergence on compacta. Let

$$
S=\left\{g \in H(D): g^{-1}(s) \in H(D) \text { or } g(s) \equiv 0\right\} .
$$

Let denote by $U$ the class of continuous functions $F: H(D) \rightarrow H(D)$ such that, for every open set $G \subset H(D)$, the intersection $\left(F^{-1} G\right) \cap S$ is non-empty.

Theorem 3. Suppose that the number $\exp \left\{\frac{2 \pi k}{h}\right\}$ is irrational for all $k \in \mathbb{Z} \backslash\{0\}$, and that $F \in U$. Let $K \subset D$ be a compact subset with connected complement, and $f(s)$ be a continuous function on $K$ and analytic in the interior of $K$. Then, for every $\varepsilon>0$,

$$
\liminf _{N \rightarrow \infty} \frac{1}{N+1} \sharp\left\{0 \leq m \leq N: \sup _{s \in K}|F(\zeta(s+i m h))-f(s)|<\varepsilon\right\}>0 .
$$

We note, that the statement of Theorem 3 is theoretical, it is difficult to check the hypothesis of the class $U$. We will give a simplification of the class $U$. Let denote by $U_{1}$ the class of continuous functions $F: H(D) \rightarrow H(D)$ such that, for each polynomial $p=p(s)$, the intersection $\left(F^{-1}\{p\}\right) \cap S$ is non-empty.

Theorem 4. Suppose that the number $h$, set $K$ and function $f(s)$ satisfy the hypotheses of Theorem 3, and that $F \in U_{1}$. Then the assertion of Theorem 3 is true.

The condition of the class $U_{1}$ is related to the non-vanishing of the preimage $F^{-1}\{p\}$ for each polynomial $p$. Obviously, if the absolute value of the constant term of a polynomial is sufficiently large, then this polynomial has no roots in a bounded region. This observation leads to the following simplification of Theorem 4.

Let $V$ be an arbitrary positive number. Define

$$
D_{V}=\{s \in \mathbb{C}: 1 / 2<\sigma<1,|t|<V\}
$$

and $S_{V}=\left\{g \in H\left(D_{V}\right): g^{-1}(s) \in H\left(D_{V}\right)\right.$ or $\left.g(s) \equiv 0\right\}$. Let consider the class $U_{1, V}$ of continuous functions $F: H\left(D_{V}\right) \rightarrow H\left(D_{V}\right)$ such that, for each polynomial $p=p(s)$, the intersection $\left(F^{-1}\{p\}\right) \cap S_{V}$ is non-empty.

Theorem 5. Suppose that the number $h$, set $K$ and function $f(s)$ satisfy the hypotheses of Theorem 3 , and $V>0$ is such that $K \subset D_{V}$. If $F \in U_{1, V}$, then the assertion of Theorem 3 is true. 
We give an example. Let

$$
F(g)=c_{1} g^{\prime}+\cdots+c_{r} g^{(r)}, \quad g \in H\left(D_{V}\right), c_{1}, \ldots, c_{r} \in \mathbb{C} \backslash\{0\} .
$$

In view of the integral Cauchy formula, the function $F$ is continuous. Moreover, it is easy to check that, for every polynomial $p=p(s)$, there exists a polynomial $q=q(s)$ such that $q \in F^{-1}\{p\}$ and $q(s) \neq 0$ for $s \in D_{V}$. Then, by Theorem 5 , the function $c_{1} \zeta^{\prime}(s)+\cdots+c_{r} \zeta^{(r)}(s)$ is universal in the sense of Theorem 3 .

Let define one more class of continuous functions $F: H(D) \rightarrow H(D)$. For $a_{1}, \ldots, a_{r} \in \mathbb{C}$, let

$$
H_{F(0) ; a_{1}, \ldots, a_{r}}(D)=\left\{g \in H(D):\left(g(s)-a_{j}\right)^{-1} \in H(D), j=1, \ldots, r\right\} \cup\{F(0)\} .
$$

Let denote by $U_{a_{1}, \ldots, a_{r}}$ the class of continuous functions $F: H(D) \rightarrow H(D)$ such that $F(S) \supset H_{F(0) ; a_{1}, \ldots, a_{r}}(D)$.

Theorem 6. Suppose that $F \in U_{a_{1}, \ldots, a_{r}}$, and that the number $h$ satisfies the hypothesis of Theorem 3. For $r=1$, let the set $K$ satisfy the hypotheses of Theorem 3, and the function $f(s)$ be continuous and $\neq a_{1}$ on $K$, and analytic in the interior of $K$. For $r \geq 2$, let $K$ be an arbitrary compact subset of $D$, and $f(s) \in H_{F(0) ; a_{1}, \ldots, a_{r}}(D)$. Then the assertion of Theorem 3 is true.

It follows from Theorem 6 that, for the functions $\zeta^{N}(s), N \in \mathbb{N},(r=1$, $\left.a_{1}=0\right)$ and $\sin (\zeta(s)), \cos (\zeta(s)), \sinh (\zeta(s)), \cosh (\zeta(s)),\left(r=2, a_{1}=-1\right.$, $\left.a_{2}=1\right)$ the assertion of Theorem 3 holds.

\section{Limit Theorems}

In this section, we present probabilistic limit theorems which are applied for the proof of universality. Let $\mathfrak{B}(X)$ be the class of Borel sets of the space $X$ and let define

$$
\Omega=\prod_{p} \gamma_{p}
$$

where $\gamma_{p}=\{s \in \mathbb{C}:|s|=1\}$ for all primes $p$. By the Tikhonov theorem, the infinite-dimensional torus $\Omega$ with the product topology and pointwise multiplication is a compact topological Abelian group. Therefore, on $(\Omega, \mathfrak{B}(\Omega))$, the unique probability Haar measure $m_{H}$ exists. This gives the probability space $\left(\Omega, \mathfrak{B}(\Omega), m_{H}\right)$. Let denote by $\omega(p)$ the projection of $\omega \in \Omega$ to the coordinate space $\gamma_{p}$, and on $\left(\Omega, \mathfrak{B}(\Omega), m_{H}\right)$, define the $H(D)$ - valued random element $\zeta(s, \omega)$ by the formula

$$
\zeta(s, \omega)=\prod_{p}\left(1-\frac{\omega(p)}{p^{s}}\right)^{-1} .
$$

We note that the latter product, for almost all $\omega \in \Omega$ with respect to the measure $m_{H}$, converges uniformly on compact subsets of the strip $D$. All above statements can be found in [6]. Let $P_{\zeta}$ be the distribution of the random element $\zeta(s, \omega)$, i.e.,

$$
P_{\zeta}(A)=m_{H}(\omega \in \Omega: \zeta(s, \omega) \in A), \quad A \in \mathfrak{B}(H(D)) .
$$


Lemma 1. Suppose that the number $\exp \left\{\frac{2 \pi k}{h}\right\}$ is irrational for all $k \in \mathbb{Z} \backslash\{0\}$. Then the probability measure

$$
P_{N}(A) \stackrel{\text { def }}{=} \frac{1}{N+1} \sharp\{0 \leq m \leq N: \zeta(s+i m h) \in A\}, \quad A \in \mathfrak{B}(H(D))
$$

converges weakly to $P_{\zeta}$ as $N \rightarrow \infty$.

Proof. The assertion of the lemma is known, however, its direct proof is not fixed in literature. The proof runs with obvious changes in the same way as that of a continuous limit theorem [6] for the measure

$$
\frac{1}{T} \operatorname{meas}\{\tau \in[0, T]: \zeta(s+i \tau) \in A\}, \quad A \in \mathfrak{B}(H(D)),
$$

as $T \rightarrow \infty$. On the other hand, Lemma 1 is a corollary of an analogous theorem from [5] obtained for the Matsumoto zeta-function, since the Riemann zetafunction is a particular case of the Matsumoto zeta-function.

In the sequel, we will use several times the following well-known fact. Let $X_{1}$ and $X_{2}$ be two metric spaces, and $u: X_{1} \rightarrow X_{2}$ be a $\left(\mathfrak{B}\left(X_{1}\right), \mathfrak{B}\left(X_{2}\right)\right)$ - measurable function. Then every probability measure $P$ on $\left(X_{1}, \mathfrak{B}\left(X_{1}\right)\right)$ induces on $\left(X_{2}, \mathfrak{B}\left(X_{2}\right)\right)$ the unique probability measure $P u^{-1}$ defined by the formula

$$
P u^{-1}(A)=P\left(u^{-1} A\right), \quad A \in \mathfrak{B}\left(X_{2}\right) .
$$

Lemma 2. Let $P_{n}, n \in \mathbb{N}$, and $P$ be probability measures on $\left(X_{1}, \mathfrak{B}\left(X_{1}\right)\right)$, and $u: X_{1} \rightarrow X_{2}$ be a continuous function. Suppose that $P_{n}$ converges weakly to $P$ as $n \rightarrow \infty$. Then $P_{n} u^{-1}$ also converges weakly to $P u^{-1}$ as $n \rightarrow \infty$.

The lemma is a particular case of Theorem 5.1 from [3].

Lemma 3. Suppose that $F: H(D) \rightarrow H(D)$ is a continuous function, and that the number $h$ is the same as in Lemma 1 . Then the probability measure

$$
P_{N, F}(A) \stackrel{\text { def }}{=} \frac{1}{N+1} \sharp\{0 \leq m \leq N: F(\zeta(s+i m h)) \in A\}, \quad A \in \mathfrak{B}(H(D)),
$$

converges weakly to the distribution of the random element $F(\zeta(s, \omega))$ as $N \rightarrow \infty$.

Proof. The lemma is an immediate corollary of Lemmas 1 and 2. Really, we have that $P_{N, F}=P_{N} F^{-1}$. Since the function $F$ is continuous, Lemmas 1 and 2 imply the weak convergence of the measure $P_{N, F}$ to $P_{\zeta} F^{-1}$ as $N \rightarrow \infty$. However, since

$$
\begin{aligned}
P_{\zeta} F^{-1}(A) & =P_{\zeta}\left(F^{-1} A\right)=m_{H}\left(\omega \in \Omega: \zeta(s, \omega) \in F^{-1} A\right) \\
& =m_{H}(\omega \in \Omega: F(\zeta(s, \omega)) \in A), \quad A \in \mathfrak{B}(H(D)),
\end{aligned}
$$

$P_{\zeta} F^{-1}$ is the distribution of the random element $F(\zeta(s, \omega))$.

For $V>0$, we denote by $P_{N, V}$ and $P_{\zeta, V}$ the restrictions to the space $\left(H\left(D_{V}\right), \mathfrak{B}\left(H\left(D_{V}\right)\right)\right)$ for the measures $P_{N}$ and $P_{\zeta}$, respectively. 
Lemma 4. For every $V>0$, the measure $P_{N, V}$ converges weakly to $P_{\zeta, V}$ as $N \rightarrow \infty$.

Proof. Obviously, the function $u: H(D) \rightarrow H\left(D_{V}\right)$ given by the formula $u(g(s))=\left.g(s)\right|_{s \in D_{V}}, g \in H(D)$, is continuous. Therefore, the lemma follows from Lemmas 1 and 2.

Lemma 5. Suppose that the function $F: H\left(D_{V}\right) \rightarrow H\left(D_{V}\right)$ is continuous. Then the probability measure

$$
P_{T, F, V}(A) \stackrel{\text { def }}{=} \frac{1}{N+1} \sharp\{0 \leq m \leq N: F(\zeta(s+i m h)) \in A\}, \quad A \in \mathfrak{B}\left(H\left(D_{V}\right)\right),
$$

converges weakly to the distribution of the random element $F\left(\zeta_{V}(s, \omega)\right)$.

Proof. We use Lemmas 2 and 4, and repeat the proof of Lemma 3.

\section{Supports}

In this section, we discuss the supports of the limit measures in limit theorems of Section 2. For this, we will use the properties of the classes $U, U_{1}, U_{1, V}$ and $U_{a_{1}, \ldots, a_{r}}$. We remind that if $X$ is a separable metric space, and $P$ is a probability measure on $(X, \mathfrak{B}(X))$, then a minimal closed set $S_{P}$ such that $P\left(S_{P}\right)=1$ is called the support of the measure $P$. The support of the distribution of a random element is called the support of that element.

Lemma 6. The support of the random element $\zeta(s, \omega)$ is the set $S$.

Proof of the lemma is given in [6, Lemma 6.5.5].

Lemma 7. The support of the random element $\zeta_{V}(s, \omega)$ is the set $S_{V}$.

Proof of the lemma completely coincides with that of Lemma 6 .

Lemma 8. Suppose that $F \in U$. Then the support of the random element $F(\zeta(s, \omega))$ is the whole of $H(D)$.

Proof. Let $g$ be arbitrary element of $H(D)$, and $G$ be an open neighbourhood of $g$. Since the function $F$ is continuous, the set $F^{-1} G$ is open as well. Moreover, by the definition of the class $U$, there exists an element $g_{1} \in S$ which is also an element of $F^{-1} G$. Thus, $F^{-1} G$ is an open neighbourhood of the element $g_{1}$. However, the support of the random element $\zeta(s, \omega)$ consists of all elements $g_{1}$ such that, for every open neighbourhood $G_{1}$ of $g_{1}$, the inequality $P_{\zeta}\left(G_{1}\right)>0$ is satisfied. Therefore, by Lemma 6 ,

$$
m_{H}(\omega \in \Omega: F(\zeta(s, \omega)) \in G)=m_{H}\left(\omega \in \Omega: \zeta(s, \omega) \in F^{-1} G\right)>0 .
$$

Since $g$ and $G$ are arbitrary, this proves the lemma.

For the investigation of supports of other random elements, we will apply the Mergelyan theorem on the approximation of analytic functions by polynomials. 
Lemma 9. Let $K \subset \mathbb{C}$ be a compact subset with connected complement, and let $f(s)$ be a continuous function on $K$ which is analytic in the interior of $K$. Then, for every $\varepsilon>0$, there exists a polynomial $p(s)$ such that

$$
\sup _{s \in K}|f(s)-p(s)|<\varepsilon .
$$

Proof of the lemma can be found in $[7,12]$.

Lemma 10. Suppose that $F \in U_{1}$. Then the support of the random element $F(\zeta(s, \omega))$ is the whole of $H(D)$.

Proof. We will prove that $F \in U$. Then the lemma will follow from Lemma 8.

Let $\varepsilon$ be an arbitrary fixed number. We fix $l_{0} \in \mathbb{N}$ such that

$$
\sum_{l>l_{0}} 2^{-l}<\frac{\varepsilon}{2}
$$

Let $\left\{K_{l}: l \in \mathbb{N}\right\}$ be a sequence of compact subsets of $D$ which occur in the definition of the metric in the space $H(D)$. Suppose that, for $f, g \in H(D)$,

$$
\sup _{s \in K_{l_{0}}}|f(s)-g(s)|<\frac{\varepsilon}{2} \text {. }
$$

Then, in view of the relation $K_{l} \subset K_{l+1}, l \in \mathbb{N}$, we find that

$$
\sup _{s \in K_{l}}|f(s)-g(s)|<\frac{\varepsilon}{2}
$$

for all $l=1, \ldots, l_{0}$. Thus, in view of $(3.1)$,

$$
\rho(f, g) \leq \sum_{l=1}^{l_{0}} 2^{-l} \frac{\sup _{s \in K_{l}}|f(s)-g(s)|}{1+\sup _{s \in K_{l}}|f(s)-g(s)|}+\sum_{l>l_{0}} 2^{-l}<\varepsilon
$$

This shows that, in the space $H(D)$, the function $g$ approximates a function $f$ with a given accuracy if $g$ approximates $f$ with a suitable accuracy uniformly on $K_{l}$ for sufficiently large $l$. Clearly, the sets $K_{l}$ can be chosen to be with connected complements. Therefore, in the space $H(D)$, we can limit ourselves by uniform approximation on compact subsets with connected complements.

Let $g$ be an arbitrary element of $H(D)$, and $G$ be an open neighbourhood of $g$. Then $F^{-1} G$ is also an open set. We will prove that the set $\left(F^{-1} G\right) \cap S$ is non-empty.

Let $K \subset D$ be a compact subset with connected complement. Then, by Lemma 9 , there exists a polynomial $p=p(s)$ which approximates the function $g$ uniformly on $K$ with desired accuracy. Therefore, since $g \in G$, we may assume that $p \in G$, too. Since $F \in U_{1}$, we have that $\left(F^{-1}\{p\}\right) \cap S \neq \varnothing$. Thus, $\left(F^{-1} G\right) \cap S \neq \varnothing$, and the lemma follows from Lemma 8. 
Lemma 11. Suppose that $F \in U_{1, V}$. Then the support of the random element $F\left(\zeta_{V}(s, \omega)\right)$ is the whole of $H\left(D_{V}\right)$.

Proof. Let $g$ be an arbitrary element of $H\left(D_{V}\right)$, and $G$ is an open neighbourhood of $g$. Then the set $F^{-1} G$ is open as well. Repeating the proof of Lemma 10, we obtain that $\left(F^{-1} G\right) \cap S_{V} \neq \varnothing$. Therefore, there exists an element $g_{1} \in S_{V}$ which also belongs to $F^{-1} G$. Thus, $F^{-1} G$ is an open neighbourhood of the element $g_{1}$. Hence, using Lemma 7 , we have that

$$
m_{H}\left(\omega \in \Omega: F\left(\zeta_{V}(s, \omega)\right) \in G\right)=m_{H}\left(\omega \in \Omega: \zeta_{V}(s, \omega) \in F^{-1} G\right)>0,
$$

and the lemma is proved.

Lemma 12. Suppose that $F \in U_{a_{1}, \ldots, a_{r}}$. Then the support of the random element $F(\zeta(s, \omega))$ contains the closure of the set $H_{F(0) ; a_{1}, \ldots, a_{r}}(D)$.

Proof. By the definition of the class $U_{a_{1}, \ldots, a_{r}}$, we have that, for each element $h \in H_{F(0) ; a_{1}, \ldots, a_{r}}(D)$, there exists an element $g \in S$ such that $F(g)=h$. Therefore, for every open neighbourhood $G$ of $h$, in view of Lemma 6,

$$
m_{H}(\omega \in \Omega: F(\zeta(s, \omega)) \in G)=m_{H}\left(\omega \in \Omega: \zeta(s, \omega) \in F^{-1} G\right)>0 .
$$

This shows that $h$ is an element of the support of random element $F(\zeta(s, \omega))$. Hence, it follows that the set $H_{F(0) ; a_{1}, \ldots, a_{r}}(D)$ and its closure is a subset of the support of $F(\zeta(s, \omega))$.

\section{Proof of the Theorems}

Proof of Theorems 3-6 uses the corresponding limit theorems, explicit forms of supports of the limit measures in them, and Lemma 9.

First we remind an equivalent of the weak convergence of probability measures in terms of open sets.

Lemma 13. Let $P_{n}, n \in \mathbb{N}$, and $P$ be probability measures on $(X, \mathfrak{B}(X))$. Then $P_{n}$ converges weakly to $P$ as $n \rightarrow \infty$ if and only if, for every open set $G \subset X$,

$$
\liminf _{n \rightarrow \infty} P_{n}(G) \geq P(G) .
$$

The lemma is a part of Theorem 2.1 from [3].

Proof of Theorem 3. By Lemma 9, there exists a polynomial $p(s)$ such that

$$
\sup _{s \in K}|f(s)-p(s)|<\frac{\varepsilon}{2} .
$$

Define the set

$$
G=\left\{g \in H(D): \sup _{s \in K}|p(s)-g(s)|<\frac{\varepsilon}{2}\right\} .
$$


Let denote by $P_{\zeta, F}$ the distribution of the random element $F(\zeta(s, \omega))$. Since the set $G$ is open, Lemmas 3 and 13 imply the inequality

$$
\liminf _{N \rightarrow \infty} \frac{1}{N+1} \sharp\{0 \leq m \leq N: F(\zeta(s+i m h)) \in G\} \geq P_{\zeta, F}(G) .
$$

In virtue of Lemma 8 , the polynomial $p(s)$ is an element of the support of the random element $F(\zeta(s, \omega))$. Since $G$ is an open neighbourhood of the polynomial $p(s)$, the properties of the support imply the inequality $P_{\zeta, F}(G)>0$. This together with (4.2) shows that

$$
\liminf _{N \rightarrow \infty} \frac{1}{N+1} \sharp\left\{0 \leq m \leq N: \sup _{s \in K}|F(\zeta(s+i m h))-p(s)|<\frac{\varepsilon}{2}\right\}>0 .
$$

Hence and from (4.1) the theorem follows.

Proof of Theorem 4. We repeat the arguments of the proof of Theorem 3, and in place of Lemma 8 we apply Lemma 10.

Proof of Theorem 5. We argue with obvious changes similarly to the proof of Theorem 3, and in place of Lemmas 3 and 8 we use Lemmas 4 and 11 .

Proof of Theorem 6. We begin with the case $r=1$. Using Lemma 9, we fix a polynomial $p(s)$ such that

$$
\sup _{s \in K}|f(s)-p(s)|<\frac{\varepsilon}{4} .
$$

Since $f(s) \neq a_{1}$ on $K$, we have that $p(s) \neq a_{1}$ on $K$ as well if $\varepsilon$ is small enough. Thus, we can define a continuous branch of $\log \left(p(s)-a_{1}\right)$ which will be an analytic function in the interior of $K$. Again, in view of Lemma 9, there exists a polynomial $p_{1}(s)$ such that

$$
\sup _{s \in K}\left|p(s)-a_{1}-e^{p_{1}(s)}\right|<\frac{\varepsilon}{4} .
$$

For brevity, we put $h_{a_{1}}(s)=e^{p_{1}(s)}+a_{1}$. Then we have that $h_{a_{1}} \in H(D)$ and, obviously, $h_{a_{1}}(s) \neq a_{1}$. Therefore, by Lemma $12, h_{a_{1}}(s)$ is an element of the support of the random element $F(\zeta(s, \omega))$. Moreover, the inequalities (4.3) and (4.4) imply that

$$
\sup _{s \in K}\left|f(s)-h_{a_{1}}(s)\right|<\frac{\varepsilon}{2} \text {. }
$$

Let define the set

$$
G_{1}=\left\{g \in H(D): \sup _{s \in K}\left|h_{a_{1}}(s)-g(s)\right|<\frac{\varepsilon}{2}\right\} .
$$

Then $P_{\zeta, F}(G)>0$, thus, by Lemmas 3 and 13 ,

$$
\liminf _{T \rightarrow \infty} \frac{1}{N+1} \sharp\left\{0 \leq m \leq N: \sup _{s \in K}\left|F(\zeta(s+i m h))-h_{a_{1}}(s)\right|<\frac{\varepsilon}{2}\right\}>0 .
$$


This and (4.5) give the assertion of the theorem.

Now let $r \geq 2$. Define the set

$$
G_{2}=\left\{g \in H(D): \sup _{s \in K}|f(s)-g(s)|<\varepsilon\right\} .
$$

Since $f(s) \in H_{F(0) ; a_{1}, \ldots, a_{r}}(D)$, Lemma 12 shows that $f(s)$ is an element of the support of the random element $F(\zeta(s, \omega))$, i.e., is an element of the support of the measure $P_{\zeta, F}$. Since $G_{2}$ is an open set, hence we have that $P_{\zeta, F}(G)>0$. Therefore, Lemmas 3 and 13 give the inequality

$\liminf _{N \rightarrow \infty} \frac{1}{N+1} \sharp\left\{0 \leq m \leq N: \sup _{s \in K}|F(\zeta(s+i m h))-f(s)|<\varepsilon\right\} \geq P_{\zeta, F}\left(G_{2}\right)>0$.

The theorem is proved.

\section{References}

[1] S.M. Voronin A.A. Karatsuba. The Riemann Zeta-Function. Walter de Gruyter, Berlin, 1992.

[2] B. Bagchi. The statistical behaviour and universality properties of the riemann zeta-function and other allied dirichlet series. PhD Thesis, Indian Statistical Institute, Calcutta, 1981.

[3] P. Billingsley. Convergence of Probability Measures. Wiley, New York, 1968.

[4] J.B. Conway. Functions of One Complex Variable. Springer-Verlag, New York, Berlin, 1978.

[5] R. Kačinskaitè. A discrete limit theorem for the matsumoto zeta function in the space of analytic functions. Lith. Math. J., 4(4):344-350, 2001. http://dx.doi.org/10.1023/A:1013808504200.

[6] A. Laurinčikas. Limit Theorems for the Riemann Zeta-Function. Kluwer, Dordrecht, Boston, London, 1996.

[7] A. Laurinčikas. Universality of the Riemann zeta-function. J. Number Theory, 130:2323-2331, 2010. http://dx.doi.org/10.1016/j.jnt.2010.04.007.

[8] D.V. Pecherskii. On rearrangement of terms in functional series. Soviet Math. Dokl., 14:633-636, 1973.

[9] A. Reich. Wertverteilung von zetafunktionen. Arch. Math., 34:440-451, 1980. http://dx.doi.org/10.1007/BF01224983.

[10] J. Steuding. Value Distribution of L-Functions, volume 1877 of Lecture Notes Math. Springer-Verlag, Berlin, Heidelberg, 2007.

[11] S.M. Voronin. Theorem on the "universality" of the Riemann zeta-function. Math. USSR Izv., 9:443-453, 1975. http://dx.doi.org/10.1070/IM1975v009n03ABEH001485.

[12] J.L. Walsh. Interpolation and approximation by rational functions in the complex domain. Amer. Math. Soc. Colloq. Publ., 20, 1960. 